tcres wird nach der Erfindung mit Perubalsam behandelt, wodurch neben der Uelerführung desselben in einen keimfreien und kcimtötenden Zustand gleichzeitig danernde Geschmeidigkeit erzielt wird. z. B. werden danach Katgutfäden folgendermaßen sterilisiert: Etwa 10 Teile Perubalsam werden in etwa 90 Teilen Aether oder heißem Alkohol gelöst und der bereits zu cincın Faden gedrehte Darm je nach der Fadenstärke kürzerc oder längere Zeit, mindestens jedoch drei Wochen der Einwirkıng diescr Lösung ausgesetzt, nachdem er bereits in ungedrchtem Zustande in einer solchen Lösung vorbehandelt wurde, um auch dic Innenseite niit Sicherheit in einen sterilen Zustand überzuführen. Nach Entnahmc aus der Lösung erfolgt die Trocknung der Katgutfäden mittels ciner Tronımel in cinem auf etwa $20^{\circ} \mathrm{C}$ erwärmten Sterilisator.

D. R. P. 265433 (Firma H. Windler in Berlin) betrifft cinen Trepau wit schraubenförmig verlaufender Schueidkallte. Die in zylinderförmiger Schraubenspur verlaufende Schneidkante hat in Achsialschnitt durch das Werkzeug ein sägezahnförmiges, ungleichseitiges Profil mit riickwärts gerichteter, unterhöhlter Schneidbrust, dagegen ein siclı1förmiges Profil in dem Sehnitt senkrecht zur Achsc. (Vgl. Fig. 1.)

Ein Urethroskop für Opcrationszwceke ist dureh D. R. P 267818 (Dr. E. R. W. Frank in Berlin) geschützt. Nach der Erfindung ist an denı Urcthroskop eine von außen einstellbare Vorrichtung (drchbare Klappe o. dgl.) angcordıct, mittels welcher der Samenhügel nicderged rückt wcrdel kann, zum Zwcoke, das Aufrichten des Samenhügels und desselı Berülırung sowic die sonstiger gesunder Gewebetcilc mit dem Operationsinstrument während der Operation zu vermeiden. Dic zum Nicderdrïcken des Sanıeıhïgels dienende Vorrichtung vom Kopf des Urethroskops aus längs dessen Schaft ist verschicbbar und der auf den Samenhügel ausgeiibte Drıck durch cinc weitere Vcrstellung der Vorrichtung veränderbar.

Gegenstand des 1). R. P. 265058 (Carnes Artificial Limb. Company iu Kansas City, Missouri, V. St. A.) ist ein künstlicher Arı, bei dem durch Zug auf eine einzige Schnur D I (vgl. Fig. 2) dic Finger abwechselıd

Fig. 2.

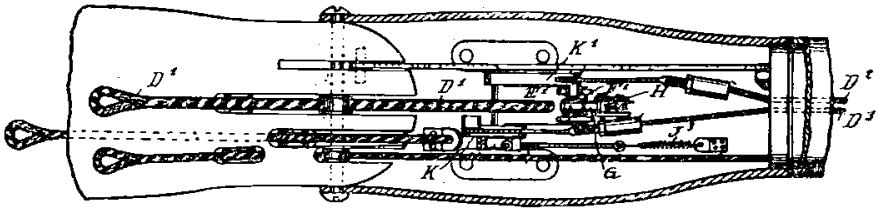

geschlossen und geöffnet wcrdell. Dic auf die Aclsse der Bctätigungs vorrichtung $T$ der Finger entgegengesetzt aufgewickeltell Schnürc $D^{2} D^{3}$ sind mit zwei Armen $K_{K^{1}}$ verbunden, die abwechselnd von einenı zwischell ihnen angeordnetell, von der gellanutell einzigen Sichnur $D^{1}$ gegen dic Wirkung einer Feder $\mathrm{J}^{3}$ ausschwingenden Hebel $\mathrm{E}^{1}$ mitgelıunneul werden, inden beiln jedesinaligen Ausschwingell des Hebels $\mathrm{E}^{1}$ durch ein Sperradgetriebe $\mathrm{G} \mathrm{G}^{1} \mathrm{H}$ abwechselıd dic eine oder die andere der in Hebcl gelagerten und dell einen oder dell anderen Arnı $\mathrm{K} \mathrm{K}^{1}$ initıchmenden Klinken $\mathrm{F} \mathrm{F}^{1}$ ilı dic wirksanle Lage bringt.

D. R. P. 267668 (Alcx. E. Block iu st. Louis, V. St. A.) bezielıt sich auf eine Plattfußeinlage nit einel biegsanıell Fußgewölbestiitze und ciner Alzahl all der Unterseite sowic an dem Fersenende der letzteren angeorductel segmentförniger Leder- oder Tuchstücke, welche taschellartige Oeffnulngeı zur Aufllahme polsterförmniger Stützcinlageı bilden. Das Wesen der Erfindung be. steht darin, da $B$ untcrhalb der taschenartigen Oeffllungen $8,9,10$ (vgl. Fig. 3 ) der PlattfuBcinlage eille lälıgsgerichtetc, gezahntc Schiene 11 und eine kcilförmige, ulit mehreren an

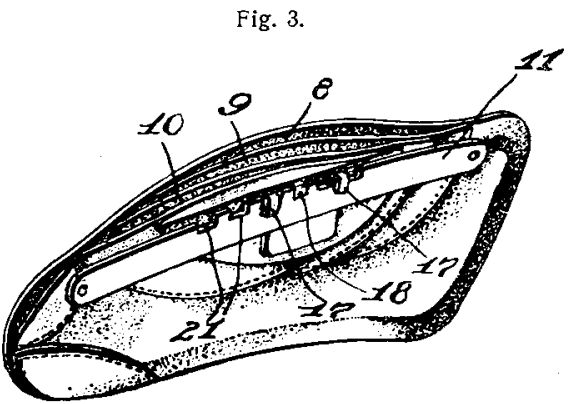

\title{
Technische Neuheiten auf dem Gebiete der Medizin, Oeffentlichen Gesundheitspflege und Krankenpflege.
}

Von Dr. M. Schall in Berlin-Grunewald.

D. R. P. 266955 (Rud. Graf in Nüirnberg) betrifft ein Verfahrell zul Herstellung von aseptiseh-sterilem Näh- und Verbandsmaterial. Letz-

1) D. In. W. $1914 \mathrm{Nr} \cdot 6$. eincr Seite der Zahuung 21 der Schiene 11 eltsprechelld angeordneten, hakeuförınigeıl Zungen 17, 18 versehene Eiılage 15 vorgesehen sind, sodab die letztere mit der gezahuten Schieıc in versehicdenen Stellungen ill Eingriff gebracht werden kann.

Gewebte oder gewirkte Gummiwaren, insbosondere für clastische Leibbinden, ließ sich die Firma Diedrich Grote Nachf. in Kempen, Rh. durch D. R. P. 267419 schützen. Nach der Erfindung sind zur Sichaffung von unclastischen oder Ucbergangsstellen die Zwischenräume zwischen den Guinmifäden in Richtung der letzteren mit festem Garn abgesteppt. 
D. R. P. 264953 (Th. Schlösser in Köln) stellt eine verstellbare Rüeken- und Koplstütze, die am Kopfende von Krankenbetten anbring-

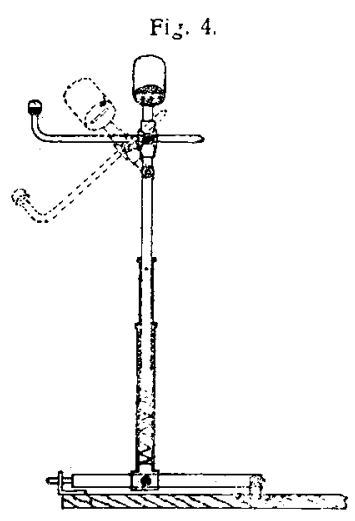
bar ist, unter Schutz. Im Gegensatz zu der bekannten Kopf- und Rückenstütze ist die neue Einrichtung an einem horizontal schwingbaren Arm befestigt, der in seiner Längsrichtung teleskopartig gegen Federdruck zusammenschiebbar eingerichtet ist, sodaß beide Stïtzen eine Parallelverschiebung ausführen können (vgl. Fig. 4).

D. R. P. 267669 (Dr. Q. Sigurini in Udine, Italien) betrifft eine Leibbinde und besteht in der Vereinigung zweier Einzelbinden, die mittels Durchsteckens, Nähten o. dgl. sich verbinden lassen oder verbunden sind und von denen bei der einen der Bauchteil, bei der anderen der Rückenteil als Hauptdruckfläche ausgebildet ist und die Verschnürungs- bzw. Ueberkreuzungsstellen entsprechend entgegengesetzt angeordnet sind.

Eine Vorrichtung zur Erzeugung gasförmiger Fmanationen der Radioelemente, bei der ein Gas unter Vermittlung eines Verteilungsorgans durch Gefäße mit Radioflüssigkeit geblasen wird, ist $H$. Kohlrausch in Zürich

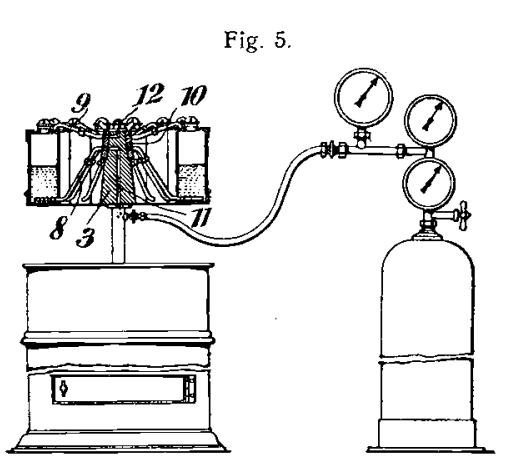
durch D. R. P. 267526 geschützt. Das neue Merkmal besteht nun darin, daß mit Radioflüssigkeit gefüllte, auf einem Drehgestell angeordnete Gefäße durch Rohrleitungen 8,9 (vgl. Fig. 5) derart mit in einem als Hahnsitz ausgebildeten, sich mit dem Drehgestell drehenden Teile 10 vorgesehenen Oeffnungen verbunden sind, daß das durch eine Boh. rung $\mathbf{I I}$ in den als Hahnkücken ausgebildeten feststehenden Drehlagerteil 3 eintretende Gas entsprechend der Drehgestellslage durch eins oder mehrere der Gefäße hindurch wieder nach dem Hahnsitz gelangt und durch eine zweite Bohrung 12 in Hahnkücken weitergeleitet wird.

Um eine Einrichtung zur Herstellung, Heizung und Trocknung von Sandbädern handelt es sich bei dem D. R. P. 265435 von Max Franke in Charlottenburg und Hackel \& Picht in Berlin. Das Wesen des Erfindungsgegenstandes besteht darin, daß die mit Taschen versehene Packung zwecks Herstellung von Sandsitzbädern in Form eines Stuhlsitzes gehalten ist. Die Packung ist mit radialen Trennungsnähten zur Herstellung strahlenförmig von der Mitte ausgehender Teilsäcke versehen, für deren Füllung in der Mitte der Packung zweckmäßig eine einzige versehließbare Oeffnung angeordnet ist. Ferner bildet die Packung die obere Abschlußfläche einer mit einer Heizquelle versehenen Heizkammer, die unterhalb der Sitzfläche eines Sitzmöbelgestelles angeordnet ist. 